ARTICLE OPEN

Check for updates

\title{
Computational search for magnetic and non-magnetic 2D topological materials using unified spin-orbit spillage screening
}

\author{
Kamal Choudhary $\mathbb{D}^{1 凶}$, Kevin F. Garrity ${ }^{1}$, Jie Jiang ${ }^{2}$, Ruth Pachter ${ }^{2}$ and Francesca Tavazza ${ }^{1}$
}

Two-dimensional topological materials (2D TMs) have a variety of properties that make them attractive for applications including spintronics and quantum computation. However, there are only a few such experimentally known materials. To help discover new 2D TMs, we develop a unified and computationally inexpensive approach to identify magnetic and non-magnetic 2D TMs, including gapped and semi-metallic topological classifications, in a high-throughput way using density functional theory-based spin-orbit spillage, Wannier-interpolation, and related techniques. We first compute the spin-orbit spillage for the $\sim 10002 \mathrm{D}$ materials in the JARVIS-DFT dataset, resulting in 122 materials with high-spillage values. Then, we use Wannier-interpolation to carry-out $Z_{2}$, Chernnumber, anomalous Hall conductivity, Curie temperature, and edge state calculations to further support the predictions. We identify various topologically non-trivial classes such as quantum spin-Hall insulators, quantum anomalous-Hall insulators, and semimetals. For a few predicted materials, we run $\mathrm{G}_{0} \mathrm{~W}_{0}+\mathrm{SOC}$ and $\mathrm{DFT}+\mathrm{U}$ calculations. We find that as we introduce many-body effects, only a few materials retain non-trivial band-topology, suggesting the importance of high-level density functional theory (DFT) methods in predicting 2D topological materials. However, as an initial step, the automated spillage screening and Wannier-approach provide useful predictions for finding new topological materials and to narrow down candidates for experimental synthesis and characterization.

npj Computational Materials (2020)6:49; https://doi.org/10.1038/s41524-020-0319-4

\section{INTRODUCTION}

In recent years, there has been a huge upsurge in topological materials research, following the predictions and observations of Dirac, Weyl, and Majorana fermions in condensed matter systems $s^{1,2}$. Several classes of topological materials have been proposed for applications in error-reduced quantum computing ${ }^{3-}$ 6 , or as high mobility and dissipationless conductors. While there have been several recent detailed screening efforts for threedimensional (3D) non-magnetic topological materials ${ }^{7-16}$, such systematic searches for two-dimensional (2D) materials are still developing ${ }^{17-19}$, especially for magnetic systems. Nevertheless, 2D materials could be more important than 3D ones because of their unique potential as miniaturized devices and their tunability via layering or functionalization ${ }^{20}$.

2D topological insulating phases can be classified into two primary types: quantum spin Hall insulators $(\mathrm{QSHI})^{21}$, which have time-reversal symmetry (TRS), and quantum anomalous Hall insulators $(\mathrm{QAHI})^{22,23}$, which lack TRS. QSHIs, characterized by a $Z_{2}$ invariant, have an insulating bulk and Dirac cone edge features. Examples include graphene, silicene ${ }^{24}$, germanene $e^{25}$, stanine ${ }^{26}$, and $1 T^{\prime}$ metal dichalcogenides ${ }^{27,28}$. Quantum anomalous Hall insulators (QAHI), characterized by a $Z$ invariant known as the Chern number, are magnetic materials with a bulk gap and quantized conducting edge channels, even in the absence of an external magnetic field. Thus far, QAHI-like behavior has been observed experimentally in very few systems: $\mathrm{Cr}, \mathrm{V}$ doped $(\mathrm{Bi}$, $\mathrm{Sb})_{2} \mathrm{Te}_{3}{ }^{22}$ and $\mathrm{MnBi}_{2} \mathrm{Te}_{4}{ }^{29-32}$, under highly controlled conditions. While there have been many theoretical works predicting QAHIs in 2D materials, surfaces, or interfaces, a systematic investigation of monolayer 2D materials is lacking. Some previously explored examples include transition metal halides such as $\mathrm{CoBr}_{2}, \mathrm{FeCl}_{3}$, $\mathrm{NiRuCl}_{6}, \mathrm{~V}_{2} \mathrm{O}_{3}, \mathrm{FeCl}_{3}$, and $\mathrm{RuCl}_{3}{ }^{33-38}$.

Compared to gapped topological systems, semimetals in twodimensions with spin-orbit coupling are relatively under-explored. For example, graphene is a well-known example of a twodimensional semimetal; however, the Dirac point in graphene is not robust to the addition of a finite amount of spin-orbit coupling ${ }^{21}$. This splitting of symmetry-protected band crossings by spin-orbit coupling occurs for most crossings protected by two-dimensional point group symmetries. Also, unlike in three dimensions, where Weyl points can occur at generic points in the Brillion zone, Weyl points will not occur generically in two dimensions without the tuning of some external parameter ${ }^{39}$. However, it is possible for non-symmorphic symmetries to protect Dirac or Weyl points in two-dimensions ${ }^{40}$, and we find several examples of this in our work. While 2D insulting topological materials are relatively easy to classify using topological indices such as $Z_{2}$ and the Chern number, finding topological semimetals is non-trivial. Hence, having a universal strategy for screening both topological insulators and semimetals is highly desirable.

In our previous work ${ }^{8}$, we discovered several classes of nonmagnetic topological 3D materials using spin-orbit spillage ${ }^{41}$ to perform the initial screening step. The spillage technique is based on comparing density functional theory (DFT) wave-functions obtained with- and without spin-orbit coupling. Materials with a high spillage value (discussed later) are candidate non-trivial materials. In this paper, we extend this approach to screen the JARVIS-DFT 2D database to search for topological insulators and semimetals, with and without TRS. The JARVIS-DFT database is a

${ }^{1}$ Materials Science and Engineering Division, National Institute of Standards and Technology, Gaithersburg, MD 20899, USA. ${ }^{2}$ Materials Directorate, Air Force Research Laboratory, Wright-Patterson Air Force Base, OH 45433, USA. ${ }^{凶}$ email: kamal.choudhary@nist.gov 


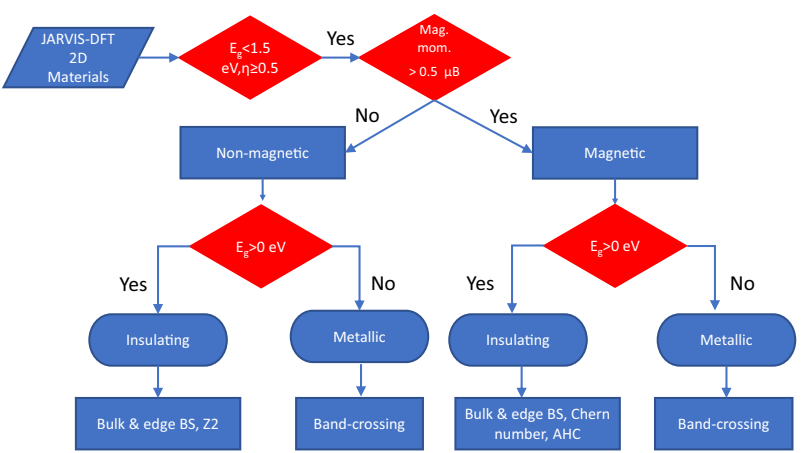

Fig. 1 Flow-chart showing the screening and analysis methodology.

part of Materials Genome Initiative (MGI) at National Institute of Standards and Technology (NIST) and contains about 40,000 3D and 1000 2D materials with their DFT-computed structural, energetics ${ }^{42}$, elastic ${ }^{43}$, optoelectronic ${ }^{43}$, thermoelectric ${ }^{44}$, piezoelectric, dielectric, infrared ${ }^{45}$, solar-efficiency ${ }^{46,47}$, and topological ${ }^{8}$ properties. Most of the 2D crystal structures we consider are derived from structures in the experimental Inorganic Crystal Structure Database (ICSD) database ${ }^{21}$, implying that most of them should be experimentally synthesizable. In this work, we screen materials from the JARVIS-DFT 2D database, searching for low bandgap materials with high-spillage ${ }^{8}$ values, identifying candidate 2D TMs. Then, for insulating compounds, we systematically carry out $Z_{2}$ calculations for non-magnetic materials and Chern number calculations for magnetic materials. For metals, we search for band crossings near the Fermi level. We also predict surface (2D) and edge (one-dimensional, 1D) band-structures, Curie temperatures, and anomalous Hall conductivity. For a subset of predicted materials, we run $\mathrm{G}_{0} \mathrm{~W}_{0}+\mathrm{SOC}$ and $\mathrm{DFT}+\mathrm{U}$ calculations. We find that as we introduce improved treatments of many-body effects, only a few materials retain a non-trivial band-topology, suggesting the importance of higher-level electronic structure methods in predicting 2D topological materials. However, as an initial step, the automated spillage screening and Wannier analysis provide useful predictions of potential topological materials, narrowing down candidate materials for experimental synthesis and characterization.

\section{RESULTS AND DISCUSSION}

As mentioned above, the spin-orbit spillage criterion is applicable for both magnetic/non-magnetic and metallic/insulating classes of materials. A flow-chart describing our computational search using the spillage as well as the traditional Wannier-based method is shown in Fig. 1. Starting from 963 2D materials in the JARVIS-DFT database, we first screen for materials with OptB88vdW bandgaps $<1.5 \mathrm{eV}$, because SOC-induced band-inversion is limited to the magnitude of SOC. This leads to 506 materials. Then we carry out spin-polarized calculations with and without SOC, and compute spillage using Eq. (1). Setting a threshold of 0.5, we find 122 materials with high-spillage. Note that for magnetic materials, we also screen in terms of the magnetic moment, selecting only cases with magnetic moment $>0.5 \mu_{\mathrm{B}}$. As a computational note, for the spin-polarized calculations, we set the initial magnetic moment to a high initial value of $6 \mu_{B}$ per atom, to search for high spin configurations. After this initial screening, we find 19 magneticinsulating, 40 magnetic-metallic, 10 non-magnetic insulating, and 53 non-magnetic metallic materials. In Table 1, we present selected examples of each class of topological materials that we consider. The full list is given on the JARVIS-DFT website (https:// www.ctcms.nist.gov/ knc6/JVASP.html) as well as in a Figshare repository, see the "Data availability" section. Clearly, metallic topological candidates outnumber insulators. Note that previous
Table 1. Examples of various classes of 2D topological materials using spillage $(\eta)$ and Wannier calculations.

\begin{tabular}{|c|c|c|c|c|c|c|c|}
\hline Formula & JID & Spg. & $E_{\mathrm{g}}(\mathrm{eV})$ & $\eta$ & $\begin{array}{l}E_{\mathrm{f}} \\
\text { (meV/ } \\
\text { atom) }\end{array}$ & TopoClass & $T_{\mathrm{c}}(\mathrm{K})$ \\
\hline $\mathrm{Bi}_{2} \mathrm{Tel}$ & 6901 & $\mathrm{P} \overline{3} \mathrm{~m} 1$ & 0.053 & 3.1 & 43.9 & QSHI & - \\
\hline Bil & 6955 & $\mathrm{C} 2 / \mathrm{m}$ & 0.145 & 3.1 & 77.7 & QSHI & - \\
\hline $\mathrm{HfTe}_{5}$ & 19987 & Pmmn & 0.074 & 0.61 & 88.3 & QSHI & - \\
\hline TalrTe $_{2}$ & 6238 & $\mathrm{P} 2_{1} / \mathrm{m}$ & 0.034 & 1.02 & 100.4 & QSHI & - \\
\hline $\mathrm{ZrFeCl}_{6}$ & 13600 & P312 & 0.011 & 1.01 & 72.0 & QAHI & 5.3 \\
\hline $\mathrm{CoBr}_{2}$ & 6034 & $\mathrm{P} \overline{3} \mathrm{~m} 1$ & 0.019 & 1.00 & 75.5 & QAHI & 17.7 \\
\hline $\mathrm{VAg}\left(\mathrm{PSe}_{3}\right)_{2}$ & 60525 & $C 2$ & 0.018 & 0.50 & 74.7 & QAHI & - \\
\hline $\mathrm{Ti}_{2} \mathrm{Te}_{2} \mathrm{P}$ & 27864 & $\mathrm{P} \overline{3} \mathrm{~m} 1$ & 0.0 & 1.06 & 58.9 & NM-Semi & - \\
\hline $\mathrm{ZrTiSe}_{4}$ & 27780 & $\mathrm{P} 2 / \mathrm{m}$ & 0.0 & 2.00 & 96.1 & NM-Semi & - \\
\hline $\mathrm{MoS}_{2}$ & 730 & $\mathrm{R} \overline{3} \mathrm{~m}$ & 0.0 & 0.98 & 89.4 & NM-Semi & - \\
\hline $\mathrm{AuTe}_{2}$ & 27775 & $\mathrm{P} \overline{3} \mathrm{~m} 1$ & 0.0 & 0.50 & 317.6 & NM-Semi & - \\
\hline $\mathrm{MnSe}$ & 14431 & $\mathrm{P} 4 / \mathrm{nmm}$ & 0.0 & 0.82 & 81.8 & M-Semi & - \\
\hline $\mathrm{FePSe}_{3}$ & 27940 & $\mathrm{P} \overline{3} \mathrm{~m} 1$ & 0.0 & 1.07 & 74.9 & M-Semi & 148.3 \\
\hline FeTe & 6667 & $\mathrm{P} 4 / \mathrm{nmm}$ & 0.0 & 1.2 & 90.2 & M-Semi & 224.7 \\
\hline $\mathrm{CoO}_{2}$ & 31379 & $\mathrm{C} 2 / \mathrm{m}$ & 0.0 & 2.01 & - & M-Semi & - \\
\hline $\mathrm{TiCl}_{3}$ & 13632 & $\mathrm{P} \overline{3} \mathrm{~m} 1$ & 0.0 & 0.62 & 72.4 & M-Semi & - \\
\hline $\mathrm{VBr}_{2} \mathrm{O}$ & 6832 & Pmmm & 0.0 & 0.45 & 67.7 & M-Semi & - \\
\hline $\mathrm{Co}(\mathrm{OH})_{2}$ & 28106 & $\mathrm{C} 2 / \mathrm{m}$ & 0.0 & 2.1 & - & M-Semi & - \\
\hline
\end{tabular}

JID: JARVIS-ID, Spg.: spacegroup, $E_{\mathrm{g}}$ : bandgap $(\mathrm{eV}), E_{\mathrm{f}}$ : exfoliation energy (meV/atom), $\eta$ : spillage, TopoClass: topological class of the material, $T_{c}$ : Curie temperature. Here, QSHI, QAHI, NM-Semi, M-Semi represents quantum spin Hall insulators, quantum anomalous Hall insulators, nonmagnetic semimetals and magnetic semi-metals, respectively.

2D topological material searches were mostly limited to insulators, but our approach can be extended to semimetals as well. Some of the predicted materials have already been experimentally synthesized, including $\mathrm{Bi}_{2} \mathrm{Tel}^{48}, \mathrm{RuCl}_{3}{ }^{49}, \mathrm{FeTe}^{50}$, etc., but the experimental confirmation of their topological properties is still ongoing.

In the remaining part of the discussion, we analyze the overall distribution of topological materials, and then we focus on individual topological classes, exploring a few examples. Information for other similar materials is provided in the JARVIS-DFT database. Finally, we discuss a few cases of DFT $+U$ and $\mathrm{G}_{0} \mathrm{~W}_{0}+\mathrm{SOC}$ calculations as a way to understand the limits of semi-local DFT calculations as screening tools for topological properties.

\section{Spin-orbit spillage analysis}

In Fig. 2 we show the spillage-based distribution for twodimensional materials. We find that 122 materials have high spillage (with a threshold of 0.5 ). The spillage criterion does, therefore, eliminate many materials, as shown in Fig. 2a. The 122 selected materials include both magnetic and non-magnetic materials, as well as both metals and insulators, and contains examples with a variety of chemical prototypes and crystal structures. The magnetic moments of topological materials range from 0 to $6 \mu \mathrm{B}$, as shown in Fig. 2b. The most common topological $2 D$ material types are $A B_{2}, A B C, A B_{3}$, and $A B$. Note that in experimental synthesis it might be easier to synthesize simple chemical prototypes such as $A B$ or 1:1 structures. Hence, having a variety of chemical prototypes provides an improved opportunity for synthesis. We find that most of the high-spillage materials belong to highly symmetric space groups, as shown in Fig. 2e. This is consistent with our previous 3D topological materials search ${ }^{8}$. A 
(a)

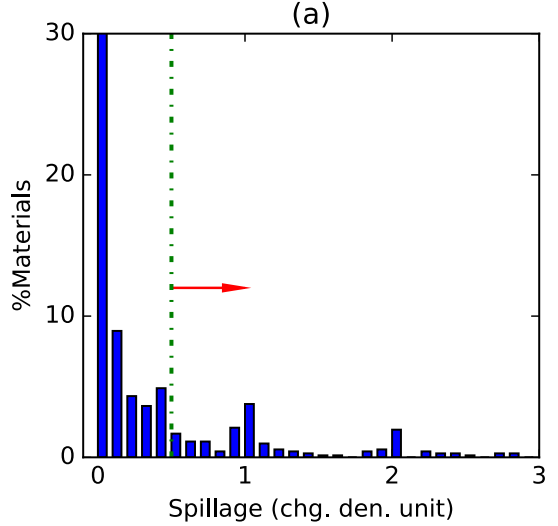

(d)

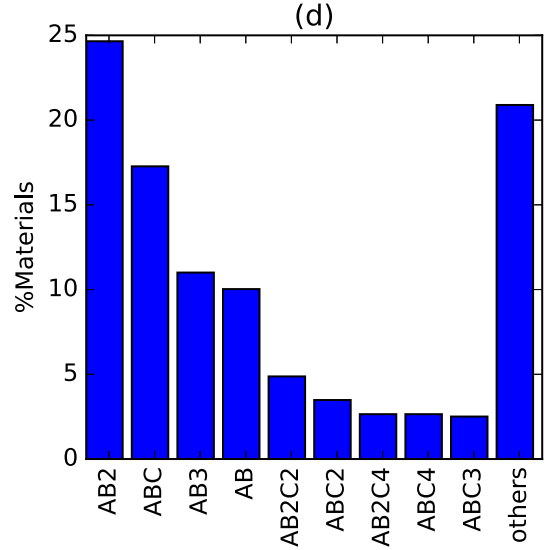

(b)

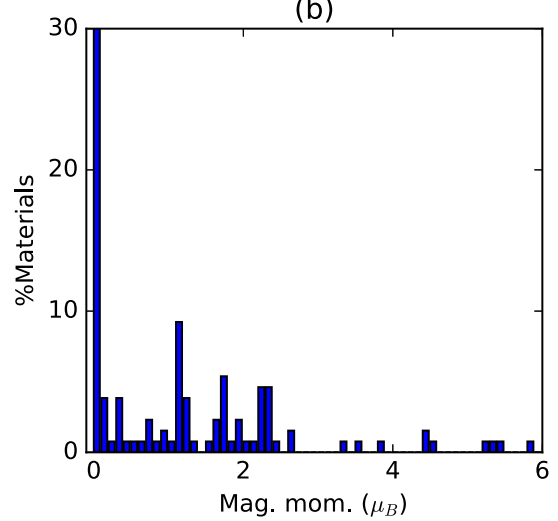

(e)

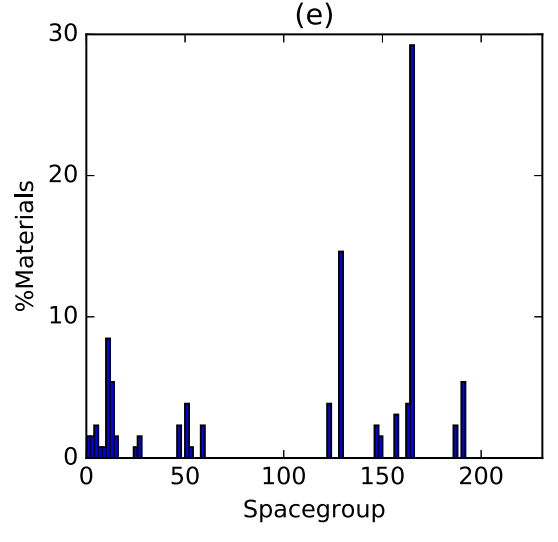

(c)

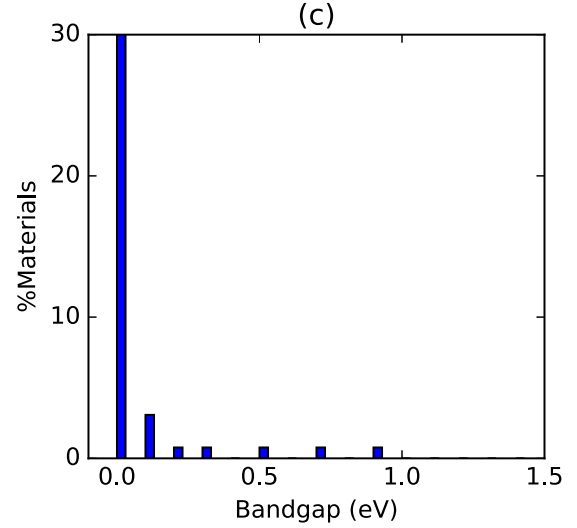

(f)

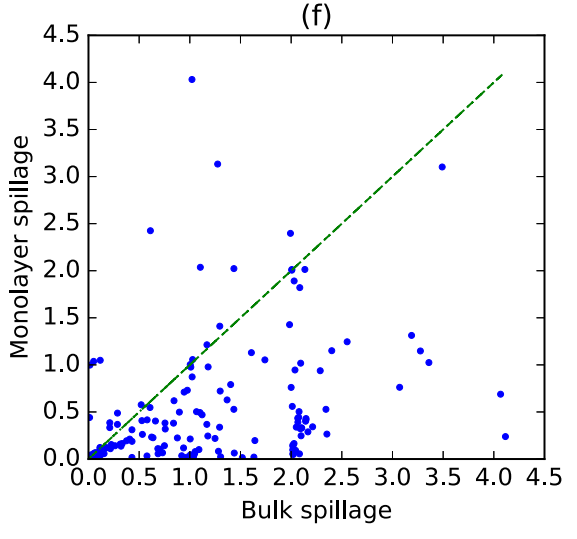

Fig. 2 Spillage assisted screening of 2D topological materials in the JARVIS-DFT database. a Spillage distribution of materials. Materials with spillage $\geq 0.5$ are likely to be topological, $\mathbf{b}$ magnetic moment distribution for high-spillage materials, $\mathbf{c}$ bandgap-distribution, $\mathbf{d}$ chemical prototype distribution; zero bandgap materials should be topological-semimetals, e space-group distribution for high spillage materials, f spillage of bulk (3D) vs monolayers (2D) for non-magnetic systems.

comparison of $3 \mathrm{D}$ and $2 \mathrm{D}$ spillage in Fig. $2 \mathrm{f}$ indicates that the 2Dmonolayer spillage values are generally lower than their 3D-bulk counterparts, but the trend is rather weak. This may be explained in part by quantum confinement in 2D-monolayers tending to increase bandgaps.

\section{Individual case studies}

Quantum spin Hall insulator (QSHI). Some of the semiconducting non-magnetic materials with high-spillage that we identified are Bil (JVASP-6955), $\mathrm{Til}_{3}$ (JVASP-6118), TalrTe 2 (JVASP-6238), $\mathrm{Bi}_{2} \mathrm{Tel}$ (JVASP-6901), and Bi (JVASP-20002). Materials such as 2D-Bi ${ }^{51}$ are experimentally known as QSHI. The fact that we find them using the spillage criteria further supports the applicability of this method to 2D materials. In order to computationally confirm that they are QSHI, we calculate the $Z_{2}$ index for a few candidates (Bil, $\left.\mathrm{Til}_{3}, \mathrm{Bi}, \mathrm{Bi}_{2} \mathrm{Tel}\right)$. We find these materials indeed have non-zero indices. We show examples of high spillage QSHI in Fig. 3. We observe high spillage peaks for all three materials (Fig. 3a, d, g), which occur near the $Z$ and Gamma points. It is difficult to observe band-inversion in Fig. $3 c$ because all the states near $E_{f}$ are derived from $\mathrm{Bi}$ orbitals, so the orbital-projected bandstructure does not show any obvious band-inversion. However, the spillage method suggests such inversion as in Fig. 3e. In all of these cases, we find that the bulk (Fig. 3b, e, h) is insulating while the edge states (Fig. $3 c, f, i)$ are conducting, with Dirac dispersions at appropriate high symmetry points, as expected for QSHI.

Quantum anomalous Hall insulator (QAHI). Next, we investigate the candidates for QAHI materials. We have 19 such candidates after screening for spillage $>0.5$, bandgap $>0.0$, magnetic moment
$>0.5$. We carry out Chern number calculations, finding several QAHI materials. Note that these were obtained using semi-local DFT calculations only, i.e., without $\mathrm{DFT}+\mathrm{U}$ or $\mathrm{G}_{0} \mathrm{~W}_{0}$ corrections; such corrections will be discussed later. Some of the materials with nonzero Chern numbers are $\mathrm{CoCl}_{2}$ (JVASP-8915), VAg(PSe $)_{2}$ (JVASP60525), and $\mathrm{ZrFeCl}_{6}$ (JVASP-13600). We show the surface and edge band-structures as well as anomalous Hall conductivity (AHC) in Fig. 4 for $\mathrm{VAg}\left(\mathrm{PSe}_{3}\right)_{2}$ and $\mathrm{ZrFeCl}_{6}$. They both have Chern number equal to 1 . Correspondingly, there is a single edge channel that connects the valence band (VB) to the conduction band (CB) (Fig. 4c, d), which carries the quantized AHC (Fig. 4d, h). Like QSHI, QAHI materials have gapped surfaces (Fig. 4b, f) but conducting edges.

Non-magnetic semimetals. Next, we identify the non-magnetic topological semimetals. This is more challenging than analyzing insulators because there are no specific $Z_{2} /$ Chern-like indices for such materials. Rather we look for Weyl/Dirac nodes between the nominal valence and conduction bands. Examples of semimetals we found and confirmed with Wannier-based methods are $\mathrm{MoS}_{2}$ (JVASP-730), AuTe 2 (JVASP-27775), $\mathrm{Ti}_{2} \mathrm{Te}_{2} \mathrm{P}$ (JVASP-27864), and $\mathrm{ZrTiSe}_{4}$ (JVASP-27780) (examples shown in Fig. 5). The complete list of found topological semimetals is given in the JARVIS-DFT website as well as in a Figshare repository, see the "Data availability" section. As is evident from Fig. 5a, c, the spillage plots have spiky peaks that represent band-inversion. For $\mathrm{Ti}_{2} \mathrm{Te}_{2} \mathrm{P}$ the band-crossing is slightly below the Fermi level while for $\mathrm{ZrTiSe}_{4}$ it is very close to the Fermi level. It is often possible to shift the Fermi-level of 2D materials via electrostatic or chemical doping, so band-crossings slightly away from Fermi-level can still have observable effects. 

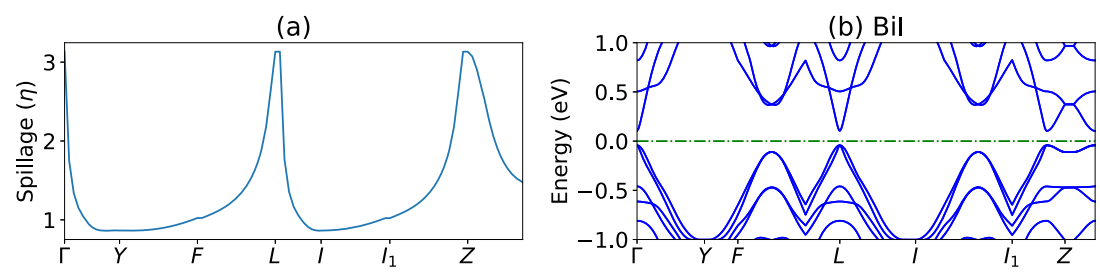

(d)
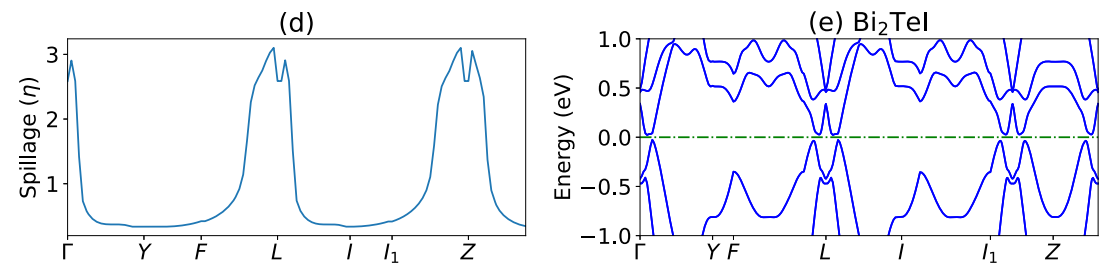

(g)
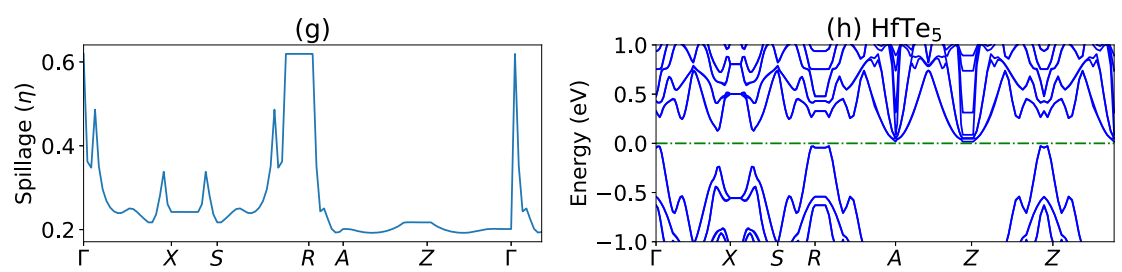
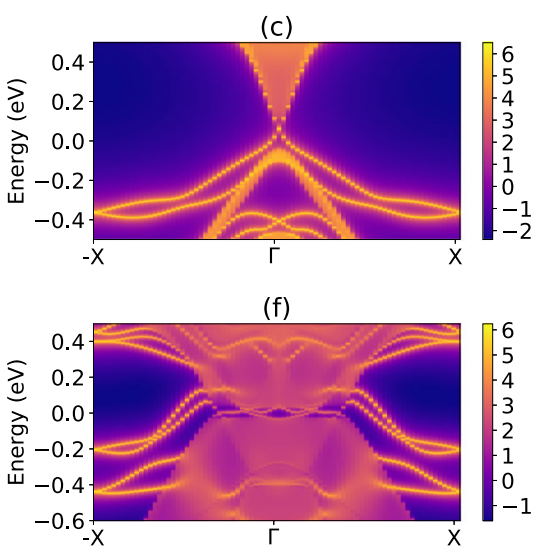

(i)

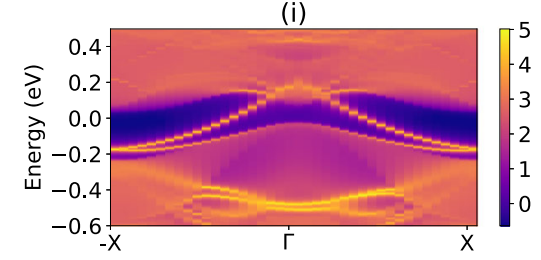

Fig. 3 Examples of quantum spin Hall insulators (QSHI). Spillage (a, d, $\mathbf{g}$ ), surface (b, e, h), and edge bandstructure (c, f, i) plots. a-c For Bil, d-f for $\mathrm{Bi}_{2} \mathrm{Tel}, \mathbf{g}-\mathbf{i}$ for $\mathrm{HfTe}_{5}$. In $(\mathbf{c}, \mathbf{f}, \mathbf{i})$ the color scale represents the electron occupation. The Fermi energy is set to zero in all band structure plots.

Magnetic semimetals. Finally, we look at ferromagnetic phases with band crossings, which are all Weyl crossings. We find band crossings near the Fermi level in MnSe (JVASP-14431), FeTe (JVASP-6667), $\mathrm{CoO}_{2}$ (JVASP-31379), $\mathrm{TaFeTe}_{3}$ (JVASP-60603), $\mathrm{TiCl}_{3}$ (JVASP-13632), and $\mathrm{Co}(\mathrm{HO})_{2}$ (JVASP-28106) all of which have nonsymmorphic symmetries that can protect band crossings ${ }^{40}$. Also, we find crossings in $\mathrm{VBr}_{2} \mathrm{O}$ (JVASP-6832), which has a double point group $(\mathrm{mmm})$ that allows two-dimensional irreducible representations. In Fig. 5e, we discuss $\mathrm{TiCl}_{3}$ (JVASP-13632), a representative magnetic semimetal. The Weyl point occurs just above the Fermi level along the high symmetry line between $\Gamma$ and $M$, as shown in Fig. $5 f$. Unfortunately, the valence band dips below the Fermi level at $\mathrm{K}$, adding extra bands to the Fermi level. Similarly, in the case of MnSe the crossings are above the Fermi-level as shown in Fig. $5 \mathrm{~g}$, h. Note that the bandgaps and magnetic moments of the above systems are heavily dependent on the calculation method. This limitation of the methodology is discussed later.

Magnetic ordering. In addition to the electronic structure, we report the magnetic ordering of the structures and Curie temperature obtained using the method described in ref. ${ }^{52}$. Many of the examples we consider have either lower energy antiferromagnetic (AFM) phases or are ferromagnetic (FM) but with spins in-plane, but for the materials we predict to be FM at finite temperature, we present their Curie temperatures and Chern numbers in Table 1 . We find that $T_{c}$ of FeTe is unusually high because of the high $J$ parameter resulting from the AFM configuration. The $\mathrm{FePS}_{3}$ and FeTe have $T_{c}$ higher than $\mathrm{Crl}_{3}$, which suggests they can be used for above liquid nitrogen temperature $T_{c}$ topological applications. On the other hand, the $T_{c}$ of $\mathrm{ZrFeCl}_{6}$ is very low, which is consistent with the large separation between $\mathrm{Fe}$ atoms in that crystal structure. As a next step, it would be interesting to study the layer dependence of $T_{c}$, which is beyond the scope of present work.

Limitations of GGA-based calculations. Next, we investigate the reliability of our calculations for a few systems using $\mathrm{GGA}+\mathrm{U}^{53,54}$ and $\mathrm{G}_{0} \mathrm{~W}_{0}+\mathrm{SOC}^{55-57}$ methods (examples shown in Fig. 6). We note that fully ab initio beyond-DFT methods like $G_{0} W_{0}$ are very time- consuming, and hence unfeasible for a high-throughput search, while $\mathrm{GGA}+\mathrm{U}$ is computationally fast, but has an adjustable parameter that limits predictive power. In Fig. $6 a$ we show the Udependence of bandgaps and Chern numbers of $\mathrm{ZrFeCl}_{6}$. We find that as we increase the $U$ parameter, $\mathrm{ZrFeCl}_{6}$ has a non-zero Chern number only for $U<0.2$. This indicates that Chern behavior can be quite sensitive to $U$. Similar behavior was also observed for $\mathrm{FeX}_{3}$ $(\mathrm{X}=\mathrm{Cl}, \mathrm{Br}, \mathrm{I})$ by $\mathrm{Li}^{35}$, where the critical value of $U$ varies from 0.43 to $0.80 \mathrm{eV}$. In Fig. $6 \mathrm{~b}$, we show how $\mathrm{G}_{0} \mathrm{~W}_{0}+\mathrm{SOC}$ can change the bandgaps for $\operatorname{VAgP}\left(\mathrm{Se}_{3}\right)_{2}$, an example QAHI. In this case, $\mathrm{G}_{0} \mathrm{~W}_{0}+\mathrm{SOC}$ increases the bandgap $(0.018 \mathrm{eV}$ vs $0.103 \mathrm{eV})$, but the band-shapes remain very similar, and the topology is nontrivial even in the $G_{0} W_{0}+S O C$ calculation. However, for the other cases, we found that the bandgap increase was substantial (Table 2 ), and caused the bands to become un-inverted, resulting in trivial materials. We note that $\mathrm{G}_{0} \mathrm{~W}_{0}+\mathrm{SOC}$ can also predict incorrect band-structures, especially for correlated transition metal compounds $^{58}$. Here we are using only single-shot $\mathrm{GW}\left(\mathrm{G}_{0} \mathrm{~W}_{0}\right)$, and more accurate results could be obtained by using fully selfconsistent (sc)- $\mathrm{GW}^{59}$, which is not carried out here due to the very high computational cost. Also, note that the bandgaps and magnetic moments can also depend on the selection of pseudopotentials. Fully accurate ab initio calculations of 2D magnetic materials remain very challenging, even for a single material, with techniques like dynamical mean field theory (DMFT) and quantum Monte-Carlo (QMC) as possible approaches for future work.

We have presented a comprehensive search of 2D topological materials, including both magnetic and non-magnetic materials, and considering insulating and metallic phases. Using the JARVISDFT 2D material dataset, we first identify materials with high spin-orbit spillage, resulting in 122 materials. Then we use Wannier-interpolation to carry-out $Z_{2}$, Chern-number, anomalous Hall conductivity, Curie temperature, and surface and edge state calculations to identify topological insulators and semimetals. For a subset of predicted QAHI materials, we run $\mathrm{G}_{0} \mathrm{~W}_{0}+\mathrm{SOC}$ and GGA $+U$ calculations. We find that as we introduce many-body effects, only a few materials retain a non-trivial band-topology, suggesting that higher-level electronic structure methods will be necessary to 
(a)

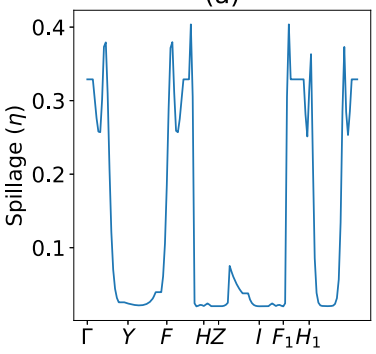

(d)

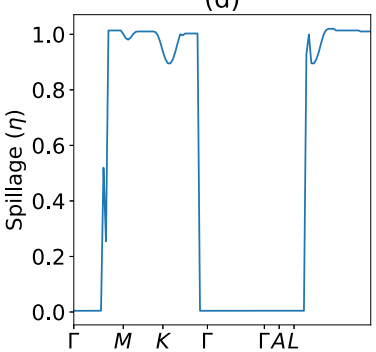

(b) $\mathrm{VAg}\left(\mathrm{PSe}_{3}\right)_{2}$

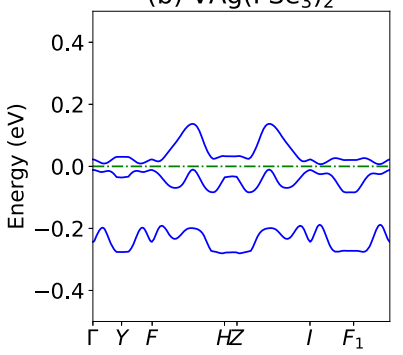

(e) $\mathrm{ZrFeCl}_{6}$

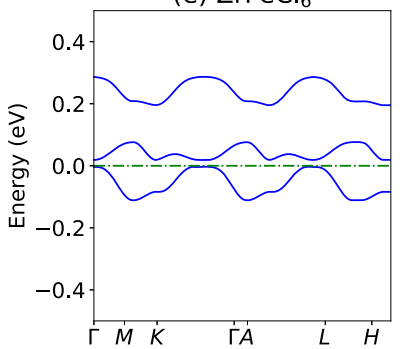

(c)

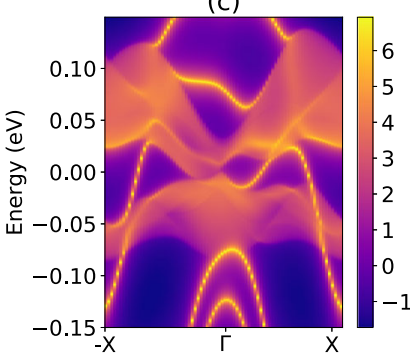

(f)

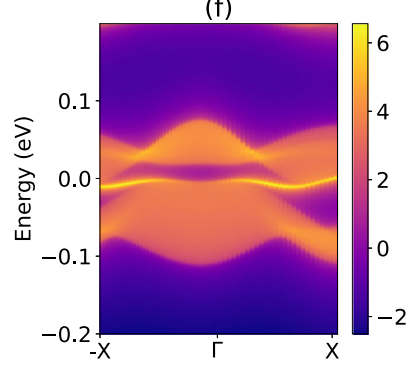

(d)

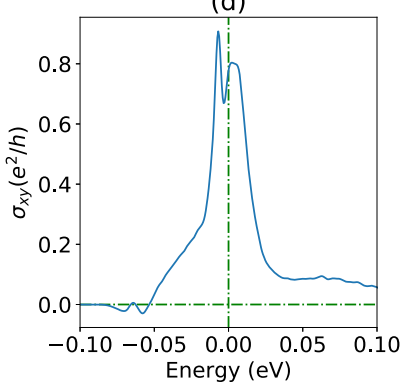

(h)

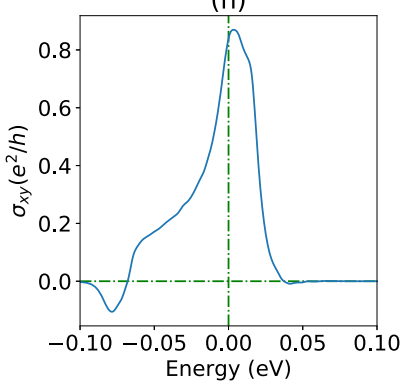

Fig. 4 Examples of quantum anomalous Hall insulators (QAHI). Spillage (a, e), surface (b, $\mathbf{f})$, and edge bandstructure (c, $\mathbf{g}$ ), and anomalous Hall conductivity (AHC) (d, h) plots. a-d For VAg $\left(\mathrm{PSe}_{3}\right)_{2}, \mathbf{e}-\mathbf{h}$ for $\mathrm{ZrFeCl}_{6}$. The Fermi energy is set to zero in all band structure plots.

fully analyze the topological properties of 2D materials. However, we believe that as an initial step, the automated spillage screening and Wannier-approach provides useful predictions for finding new topological materials.

\section{METHODS}

DFT calculations were carried out using the Vienna Ab-initio simulation package (VASP) ${ }^{60,61}$ software using the workflow given on our github page (https://github.com/usnistgov/jarvis). Please note commercial software is identified to specify procedures. Such identification does not imply recommendation by National Institute of Standards and Technology (NIST). We use the OptB88vdW functional ${ }^{59}$, which gives accurate lattice parameters for both vdW and non-vdW (3D-bulk) solids ${ }^{42}$. We optimize the crystal-structures of the bulk and monolayer phases using VASP with OptB88vdW. The initial screening step for $<1.5 \mathrm{eV}$ bandgap materials is done with OptB88vdW bandgaps from the JARVIS-DFT database. Because SOC is not currently implemented for OptB88vdW in VASP, we carry out spin-polarized PBE and spin-orbit PBE calculations in order to calculate the spillage for each material. Such an approach has been validated by refs. ${ }^{8,62}$. The crystal structure was optimized until the forces on the ions were less than $0.01 \mathrm{eV} / \AA$ and energy less than $10^{-6} \mathrm{eV}$. We also use $\mathrm{G}_{0} \mathrm{~W}_{0}+\mathrm{SOC}$ and DFT $+\mathrm{U}$ on selected structures. We use Wannier $90^{63}$ and Wannier-tools ${ }^{64}$ to perform the Wannier-based evaluation of topological invariants.

First, out of $9632 \mathrm{D}$ monolayer materials, we identify materials with bandgap $<1.5 \mathrm{eV}$, and heavy elements (atomic weight $\geq 65$ ). We calculate the exfoliation energy of a $2 \mathrm{D}$ material as the difference in energy per atom for bulk and monolayer counterparts ${ }^{42}$. Then we use the spillage technique to quickly narrow down the number of possible materials. As introduced in ref. ${ }^{41}$, we calculate the spin-orbit spillage, $\eta(\mathbf{k})$, given by the following equation:

$$
\eta(\mathbf{k})=n_{\text {occ }}(\mathbf{k})-\operatorname{Tr}(P \tilde{P})
$$

where, $P(\mathbf{k})=\sum_{n=1}^{n_{\text {occ }}(\mathbf{k})}\left|\psi_{n \mathbf{k}}\right\rangle\left\langle\psi_{n \mathbf{k}}\right|$ is the projector onto the occupied wavefunctions without SOC, and $\tilde{P}$ is the same projector with SOC for band $n$ and k-point $\mathbf{k}$. We use a $\boldsymbol{k}$-dependent occupancy $n_{\text {occ }}(\mathbf{k})$ of the non-spin-orbit calculation so that we can treat metals, which have varying

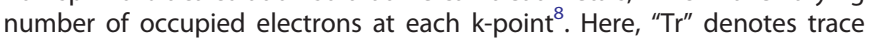
over the occupied bands. We can write the spillage equivalently as:

$\eta(\mathbf{k})=n_{\text {occ }}(\mathbf{k})-\sum_{m, n=1}^{n_{\text {occ }}(\mathbf{k})}\left|M_{m n}(\mathbf{k})\right|^{2}$ where $M_{m n}(\mathbf{k})=\left\langle\psi_{m \mathbf{k}} \mid \tilde{\psi}_{n \mathbf{k}}\right\rangle$ is the overlap between occupied Bloch functions with and without SOC at the same wave vector $\mathbf{k}$. If the SOC does not change the character of the occupied wavefunctions, the spillage will be near zero, while band inversion will result in a large spillage. In the case of insulating topological materials driven by spin-orbit-based band inversion, the spillage will be at least 1.0 for Chern insulators or 2.0 for TRSinvariant topological insulators at the k-point(s) where band inversion occurs $^{41}$. In other words, the spillage can be viewed as the number of band-inverted electrons at each k-point. For topological metals and semimetals, or cases where the inclusion of SOC opens a gap, the spillage is not required to be an integer, but we find empirically that a high spillage value is an indication of a change in the band structure due to SOC that can indicate topological behavior. We choose a threshold value of $\eta=0.5$ at any k-point for our screening procedure, based on comparison with known topological semimetals ${ }^{8}$. Our screening method can also detect topological materials with small SOC, like the small bandgap that is opened at the Dirac point in graphene ${ }^{21}$ (JVASP-667). However, the screening method is not expected to work for semimetals with topological features that are not caused or modified by spin-orbit coupling.

After spillage calculations, we run Wannier-based Chern and $Z_{2}$-index calculations for these materials.

The Chern number, $C$ is calculated over the Brillouin zone, BZ, as:

$C=\frac{1}{2 \pi} \sum_{n} \int d^{2} \mathbf{k} \Omega_{n}$

$\Omega_{n}(\mathbf{k})=-\operatorname{Im}\left\langle\nabla_{\mathbf{k}} u_{n \mathbf{k}}|\times| \nabla_{\mathbf{k}} u_{n \mathbf{k}}\right\rangle=\sum_{m \neq n} \frac{2 \operatorname{lm}\left\langle\psi_{n \mathbf{k}}\left|\hat{v}_{x}\right| \psi_{m \mathbf{k}}\right\rangle\left\langle\psi_{m \mathbf{k}}\left|\hat{v}_{y}\right| \psi_{n \mathbf{k}}\right\rangle}{\left(\omega_{m}-\omega_{n}\right)^{2}}$

Here, $\Omega_{n}$ is the Berry curvature, $u_{n \mathbf{k}}$ being the periodic part of the Bloch wave in the $n$th band, $E_{n}=\hbar \omega_{n}, v_{x}$ and $v_{y}$ are velocity operators. The Berry curvature as a function of $\mathbf{k}$ is given by:

$\Omega(\mathbf{k})=\sum_{n} \int f_{n \mathbf{k}} \Omega_{n}(\mathbf{k})$

Then, the intrinsic anomalous Hall conductivity $(\mathrm{AHC}) \sigma_{x y}$ is given by:

$\sigma_{x y}=-\frac{e^{2}}{\hbar} \int \frac{d^{3} \mathbf{k}}{(2 \pi)^{3}} \Omega(\mathbf{k})$

In addition to searching for gapped phases, we also search for Dirac and Weyl semimetals by numerically searching for band crossings between the highest occupied and lowest unoccupied band, using the algorithm from WannierTools ${ }^{64}$. This search for crossings can be performed efficiently because it takes advantage of Wannier-based band interpolation. In an ideal case, the band crossings will be the only points at the Fermi level; 
(a)

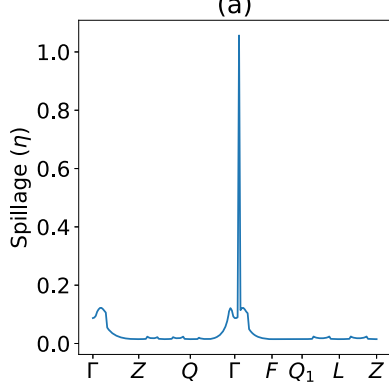

(e)

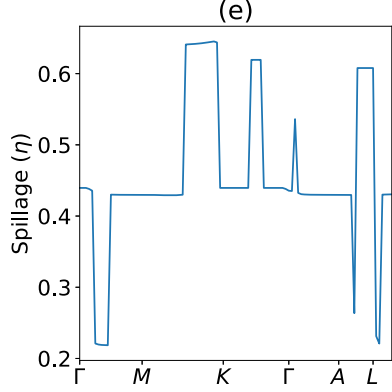

(b) $\mathrm{Ti}_{2} \mathrm{Te}_{2} \mathrm{P}$

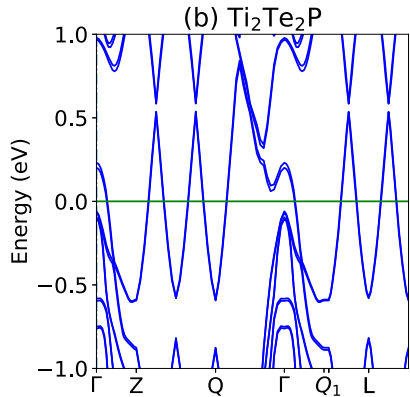

(f) $\mathrm{TiCl}_{3}$

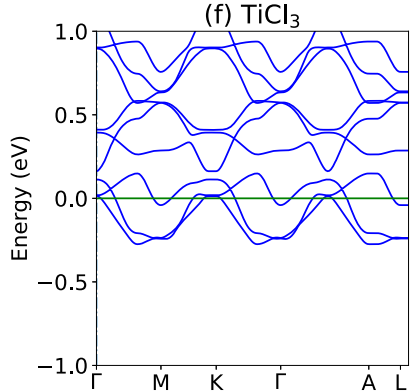

(c)

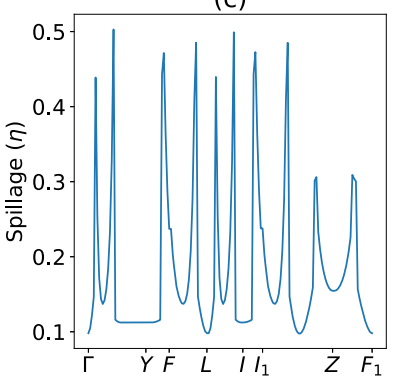

(g)

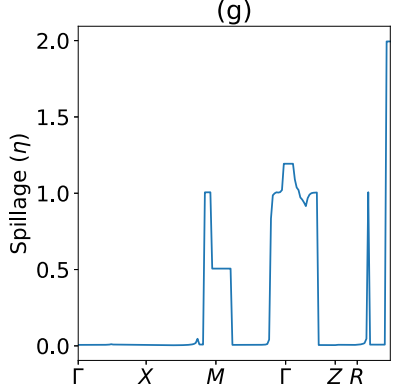

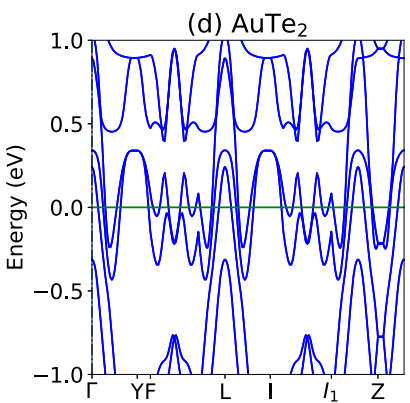

(h) $\mathrm{MnSe}$

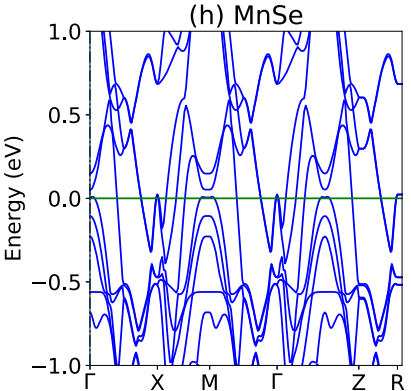

Fig. 5 Examples of topological semimetals. Non-magnetic (a-d) and magnetic (e-h) topological semimetals with spillage (a, $\mathbf{c}$, e, $\mathbf{g})$ and surface bandstructure (b, d, f, g) plots. a, b $\mathrm{Ti}_{2} \mathrm{TeP}_{1} \mathbf{c}, \mathbf{d} \mathrm{AuTe}_{2}, \mathbf{e}, \mathbf{f} \mathrm{TiCl}_{3}, \mathbf{g}, \mathbf{h} \mathrm{MnSe}$.
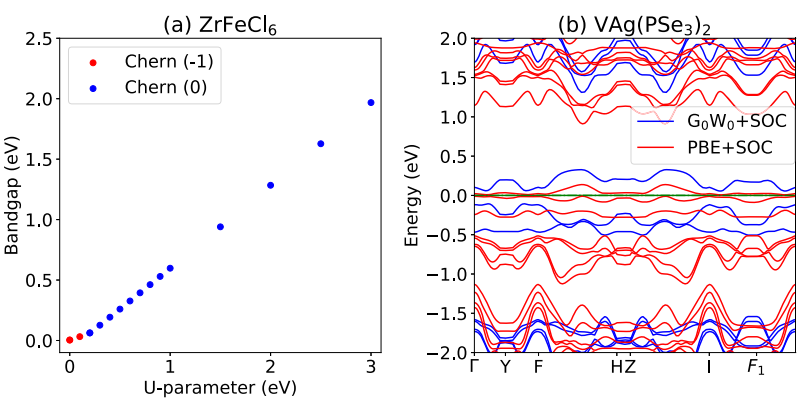

Fig. 6 Effect of beyond DFT methods on the electronic structure using $\mathbf{D F T}+\mathbf{U}$ and $\mathbf{G}_{\mathbf{0}} \mathbf{W}_{\mathbf{0}}$ methods. a $D F T+U$ effect on Chern number for $\mathrm{ZrFeCl}_{6}, \mathbf{b}$ bandstructure of $\mathrm{VAg}\left(\mathrm{PSe}_{3}\right)_{2}$ without (red) and with $\mathrm{G}_{0} \mathrm{~W}_{0}+\mathrm{SOC}$ (blue) method.

\begin{tabular}{|lcc}
\hline Table 2. & Comparison of $\mathrm{PBE}+\mathrm{SOC}$ and $\mathrm{G}_{0} \mathrm{~W}_{0}+\mathrm{SOC}$ bandgaps. \\
\hline$E_{\mathrm{g}}(\mathrm{eV})$ & $\mathrm{PBE}+\mathrm{SOC}$ & $\mathrm{G}_{0} \mathrm{~W}_{0}+\mathrm{SOC}$ \\
\hline $\mathrm{ZrFeCl}$ & 0.011 & 1.302 \\
$\left.\mathrm{VAg}_{6} \mathrm{PSe}_{3}\right)_{2}$ & 0.018 & 0.103 \\
$\mathrm{RuCl}_{3}$ & 0.0 & 0.50
\end{tabular}

however, in most cases, we find additional trivial metallic states at the Fermi level. The surface spectrum was calculated by using the Wannier functions and the iterative Green's function method ${ }^{65,66}$.

For magnetic systems, we primarily screen the ferromagnetic phase, with spins oriented out of the plane, which we expect is possible to achieve experimentally in most cases, using an external field if necessary. We initialize the magnetic moment with $6 \mu_{\mathrm{B}}$ for spin-polarized calculations. For a subset of interesting compounds, we perform a set of three additional calculations: ferromagnetic with spins in-plane, and antiferromagnetic with spins in- and out-of-plane. With these energies, we can fit a minimal magnetic model and calculate an estimated Curie temperature for ferromagnetic materials with out-of-plane spins, using the method of ref. ${ }^{52}$, which takes into account exchange constants and magnetic anisotropy. Magnetic anisotropy that favors out-of-plane spins is crucial to enable long-range magnetic ordering in two-dimensions following the Mermin-Wagner theorem ${ }^{67}$. We consider a simple Heisenberg model Hamiltonian with nearest-neighbor exchange interactions $J$, single-ion anisotropy $A$, and nearest neighbor anisotropic exchange $B$ :

$H=-\frac{1}{2} \sum_{i j} J_{i j} \boldsymbol{S}_{i} \boldsymbol{S}_{j}-A \sum_{j}\left(S_{i}^{z}\right)^{2}-\frac{1}{2} \sum_{i j} B_{i j} S_{i}^{z} S_{j}^{z}$

with $J_{i j}, A, B_{i j}>0$. The sums run over all magnetic sites and $J_{i j}=J, B_{i j}=B$ if $i$ and $j$ are nearest neighbors and zero otherwise. The maximum value of $S_{i}^{z}$ denoted by $S$. For an Ising model, which corresponds to the limit of $A \rightarrow \infty$, the critical transition temperature is given by:

$T c^{l s i n g}=\frac{S^{2} \widetilde{T C}}{k B}$

where $\widetilde{T c}$ is a dimensionless critical temperature with values of $1.52,2.27$, 2.27, and 3.64 for the honeycomb, quadratic, Kagomé, and hexagonal lattices respectively. However, systems with finite $A$, we instead use ${ }^{52}$ :

$T_{c}=T_{c}^{\operatorname{ling}^{\sin }} f\left(\frac{\Delta}{J(2 S-1)}\right)$

with $\Delta=A(2 S-1)+B S N_{n n}$ and $f(x)=\tanh ^{\frac{1}{4}}\left[\frac{6}{N_{n n}} \log (1+\gamma \mathrm{x})\right]$.

To better account for correlation effects and self-interaction error in describing transition metal elements, we apply the DFT $+U$ method to a subset of materials ${ }^{53,54}$. For a few systems, we also carry out a systematic $\mathrm{U}$-scan by varying the $U$ parameter from 0 to $3 \mathrm{eV}$ and monitor changes in the band structure ${ }^{68}$. We also perform $\mathrm{G}_{0} \mathrm{~W}_{0}+\mathrm{SOC}^{55-57}$ calculations with an ENCUTGW parameter (energy cutoff for response function) of $333.3 \mathrm{eV}$ for a few materials to analyze the impact of many-body effects.

\section{DATA AVAILABILITY}

The electronic structure data is made available at the JARVIS-DFT website: https:// www.ctcms.nist.gov/ knc6/JVASP.html and http://jarvis.nist.gov. The dataset is also available at the Figshare link: https://doi.org/10.6084/m9.figshare.12009372.

\section{CODE AVAILABILITY}

Python-language based codes with examples for calculating spillage and carrying out calculations are given at https://github.com/usnistgov/jarvis. 
Received: 23 December 2019; Accepted: 31 March 2020;

Published online: 07 May 2020

\section{REFERENCES}

1. Ren, Y., Qiao, Z. \& Niu, Q. Topological phases in two-dimensional materials: a review. Rep. Prog. Phys. 79, 066501 (2016).

2. Yan, B. \& Zhang, S.-C. Topological materials. Rep. Prog. Phys. 75, 096501 (2012).

3. Laughlin, R. B. Quantized Hall conductivity in two dimensions. Phys. Rev. B 23, 5632 (1981).

4. Thouless, D. J., Kohmoto, M., Nightingale, M. P. \& den Nijs, M. Quantized Hall conductance in a two-dimensional periodic potential. Phys. Rev. Lett. 49, 405 (1982).

5. Freedman, M., Kitaev, A., Larsen, M. \& Wang, Z. Topological quantum computation. Bull. Am. Math. Soc. 40, 31-38 (2003).

6. Sato, M. \& Ando, Y. Topological superconductors: a review. Rep. Prog. Phys. 80 , 076501 (2017).

7. Po, H. C., Vishwanath, A. \& Watanabe, H. Symmetry-based indicators of band topology in the 230 space groups. Nat. Commun. 8, 50 (2017).

8. Choudhary, K., Garrity, K. F. \& Tavazza, F. High-throughput discovery of topologically non-trivial materials using spin-orbit spillage. Sci. Rep. 9, 8534 (2019).

9. Slager, R.-J., Mesaros, A., Juričić, V. \& Zaanen, J. The space group classification of topological band-insulators. Nat. Phys. 9, 98 (2013).

10. Bradlyn, B. et al. Topological quantum chemistry. Nature 547, 298 (2017).

11. Tang, F., Po, H. C., Vishwanath, A. \& Wan, X. Towards ideal topological materials: comprehensive database searches using symmetry indicators. Nature 566, 486 (2019).

12. Tang, F., Po, H. C., Vishwanath, A. \& Wan, X. Topological materials discovery by large-order symmetry indicators. Sci. Adv. 5, 3 (2018).

13. Tang, F., Po, H. C., Vishwanath, A. \& Wan, X. Efficient topological materials dis covery using symmetry indicators. Nat. Phys. 15, 470 (2019).

14. Zhou, $X$. et al. Topological crystalline insulator states in the $\mathrm{Ca}_{2} \mathrm{As}$ family. Phys. Rev. B 98, 241104(R) (2018).

15. Vergniory, M., Elcoro, L., Felser, C., Bernevig, B. \& Wang, Z. The (high quality) topological materials in the world. Nature 566, 480 (2019).

16. Zhang, T. et al. Catalogue of topological electronic materials. Nature 566, 475 (2019).

17. Olsen, T. et al. Discovering two-dimensional topological insulators from highthroughput computations. Phys. Rev. Mater. 3, 024005 (2019)

18. Marrazzo, A., Gibertini, M., Campi, D., Mounet, N. \& Marzari, N. Abundance of $Z_{2}$ topological order in exfoliable two-dimensional insulators. Nano Lett. 19, 8431 (2019).

19. Wang, D. et al. Two-dimensional topological materials discovery by symmetryindicator method. Phys. Rev. B 100, 195108 (2019).

20. Lu, Y. Two-dimensional Materials in Nanophotonics: Developments, Devices, and Applications (CRC Press, 2019).

21. Kane, C. L. \& Mele, E. J. Quantum spin Hall effect in graphene. Phys. Rev. Lett. 95 226801 (2005).

22. Chang, C.-Z. et al. Experimental observation of the quantum anomalous Hall effect in a magnetic topological insulator. Science 340, 167-170 (2013).

23. He, K., Wang, Y. \& Xue, Q.-K. Topological materials: quantum anomalous Hall system. Annu. Rev. Condens. Matter Phys. 9, 329-344 (2018).

24. Ezawa, M. Valley-polarized metals and quantum anomalous Hall effect in silicene. Phys. Rev. Lett. 109, 055502 (2012).

25. Zhang, L. et al. Structural and electronic properties of germanene on $\mathrm{MoS}_{2}$. Phys. Rev. Lett. 116, 256804 (2016).

26. Lee, G.-H. et al. Flexible and transparent $\mathrm{MoS}_{2}$ field-effect transistors on hexagonal boron nitride-graphene heterostructures. ACS Nano 7, 7931-7936 (2013).

27. Qian, X., Liu, J., Fu, L. \& Li, J. Quantum spin Hall effect in two-dimensional transition metal dichalcogenides. Science 346, 1344-1347 (2014).

28. Tang, S. et al. Quantum spin Hall state in monolayer $1 T^{\prime}-W T e_{2}$. Nat. Phys. 13, 683 (2017).

29. Otrokov, M. M. et al. Highly-ordered wide bandgap materials for quantized anomalous Hall and magnetoelectric effects. 2D Mater. 4, 025082 (2017).

30. $\mathrm{Li}$, J. et al. Intrinsic magnetic topological insulators in van der Waals layered $\mathrm{MnBi}_{2} \mathrm{Te}_{4}$-family materials. Sci. Adv. 5, eaaw5685 (2019).

31. Liu, C. et al. Quantum phase transition from axion insulator to Chern insulator in $\mathrm{MnBi}_{2} \mathrm{Te}_{4}$. Nat. Mater. https://doi.org/10.1038/s41563-019-0573-3 (2020).

32. Deng, Y. et al. Magnetic-field-induced quantized anomalous Hall effect in intrinsic magnetic topological insulator $\mathrm{MnBi}_{2} \mathrm{Te}_{4}$. Science 367, 895-900 (2020).
33. Chen, P., Zou, J.-Y. \& Liu, B.-G. Intrinsic ferromagnetism and quantum anomalous Hall effect in a $\mathrm{CoBr}_{2}$ monolayer. Phys. Chem. Chem. Phys. 19, 13432-13437 (2017).

34. Zhang, S.-H. \& Liu, B.-G. Intrinsic 2D ferromagnetism, quantum anomalous Hall conductivity, and fully-spin-polarized edge states of $\mathrm{FeBr}_{3}$ monolayer. Preprint at: arXiv:1706.08943 (2017)

35. Li, P. Prediction of intrinsic two dimensional ferromagnetism realized quantum anomalous Hall effect. Phys. Chem. Chem. Phys. 21, 6712-6717 (2019).

36. Zhou, P., Sun, C. \& Sun, L. Two dimensional antiferromagnetic chern insulator:

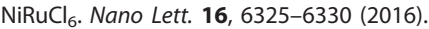

37. Wang, H., Luo, W. \& Xiang, H. Prediction of high-temperature quantum anomalous Hall effect in two-dimensional transition-metal oxides. Phys. Rev. B 95 125430 (2017)

38. Liu, H., Sun, J.-T., Liu, M. \& Meng, S. Screening magnetic two-dimensional atomic crystals with nontrivial electronic topology. J. Phys. Chem. Lett. 9, 6709-6715 (2018).

39. Garrity, K. F. \& Vanderbilt, D. Chern insulators from heavy atoms on magnetic substrates. Phys. Rev. Lett. 110, 116802 (2013).

40. Young, S. M. \& Kane, C. L. Dirac semimetals in two dimensions. Phys. Rev. Lett 115,126803 (2015).

41. Choudhary, K., Kalish, I., Beams, R. \& Tavazza, F. High-throughput identification and characterization of two-dimensional materials using density functional theory. Sci. Rep. 7, 5179 (2017)

42. Choudhary, K., Cheon, G., Reed, E. \& Tavazza, F. Elastic properties of bulk and lowdimensional materials using van der Waals density functional. Phys. Rev. B 98 014107 (2018).

43. Choudhary, K. et al. Computational screening of high-performance optoelectronic materials using OptB88vdW and TB-mBJ formalisms. Sci. Data 5, 180082 (2018).

44. Choudhary, K., Garrity, K. \& Tavazza, F. Data-driven discovery of 3D and 2D thermoelectric materials. Preprint at: arXiv:1906.06024 (2019).

45. Choudhary, K. et al. High-throughput density functional perturbation theory and machine learning predictions of infrared, piezoelectric and dielectric responses. Preprint at: arXiv:1910.01183 (2019)

46. Choudhary, K. et al. Accelerated discovery of efficient solar cell materials using quantum and machine-learning methods. Chem. Mater. 31(15), 5900 (2019)

47. Choudhary, K., DeCost, B. \& Tavazza, F. Machine learning with force-field inspired descriptors for materials: fast screening and mapping energy landscape. Phys. Rev. Mater. 2, 083801 (2018).

48. Rusinov, I. et al. Mirror-symmetry protected non-TRIM surface state in the weak topological insulator $\mathrm{Bi}_{2}$ Tel. Sci. Rep. 6, 20734 (2016).

49. Do, S.-H. et al. Majorana fermions in the Kitaev quantum spin system a- $\mathrm{RuCl}_{3}$. Nat Phys. 13, 1079 (2017).

50. Uchida, E. \& Kondoh, H. Magnetic properties of FeTe. J. Phys. Soc. Jpn. 10 357-362 (1955).

51. Jin, K.-H. \& JhiS.-H. Quantum anomalous Hall and quantum spin-Hall phases in flattened Bi and Sb bilayers. Sci. Rep. 5, 8426 (2015).

52. Torelli, D. \& Olsen, T. Calculating critical temperatures for ferromagnetic order in two-dimensional materials. 2D Mater. 6, 015028 (2018).

53. Anisimov, V. I., Zaanen, J. \& Andersen, O. K. Band theory and Mott insulators: Hubbard U instead of Stoner I. Phys. Rev. B 44, 943 (1991).

54. Dudarev, S., Botton, G., Savrasov, S., Humphreys, C. \& Sutton, A. Electron-energyloss spectra and the structural stability of nickel oxide: an LSDA+U study. Phys. Rev. B 57, 1505 (1998).

55. Hedin, L. New method for calculating the one-particle Green's function with application to the electron-gas problem. Phys. Rev. 139, A796 (1965).

56. Shishkin, M. \& Kresse, G. Implementation and performance of the frequency dependent GW method within the PAW framework. Phys. Rev. B 74, 035101 (2006).

57. Shishkin, M. \& Kresse, G. Self-consistent GW calculations for semiconductors and insulators. Phys. Rev. B 75, 235102 (2007).

58. Van Setten, M., Giantomassi, M., Gonze, X., Rignanese, G.-M. \& Hautier, G. Automation methodologies and large-scale validation for GW: towards highthroughput GW calculations. Phys. Rev. B 96, 155207 (2017).

59. Klimeš, J., Bowler, D. R. \& Michaelides, A. J. Chemical accuracy for the van der Waals density functional. J. Phys.: Condens. Matter 22, 022201 (2009).

60. Kresse, G. \& Furthmüller, J. Efficient iterative schemes for ab initio total-energy calculations using a plane-wave basis set. Phys. Rev. B 54, 11169 (1996).

61. Kresse, G. \& Furthmüller, J. Efficiency of ab-initio total energy calculations for metals and semiconductors using a plane-wave basis set. Comput. Mater. Sci. 6 , 15-50 (1996).

62. Cao, G. et al. Rhombohedral $\mathrm{Sb}_{2} \mathrm{Se}_{3}$ as an intrinsic topological insulator due to strong van der Waals interlayer coupling. Phys. Rev. B 97, 075147 (2018). 
63. Mostofi, A. A. et al. wannier90: A tool for obtaining maximally-localised Wannier functions. Comput. Phys. Commun. 178, 685-699 (2008).

64. Wu, Q., Zhang, S., Song, H.-F., Troyer, M. \& Soluyanov, A. A. WannierTools: an open-source software package for novel topological materials. Comput. Phys. Commun. 224, 405-416 (2018).

65. Marzari, N. \& Vanderbilt, D. Maximally localized generalized Wannier functions for composite energy bands. Phys. Rev. B 56, 12847 (1997).

66. Souza, I., Marzari, N. \& Vanderbilt, D. Maximally localized Wannier functions for entangled energy bands. Phys. Rev. B 65, 035109 (2001).

67. Mermin, N. D. \& Wagner, H. Absence of ferromagnetism or antiferromagnetism in one-or two-dimensional isotropic Heisenberg models. Phys. Rev. Lett. 17, 1133 (1966).

68. Stevanović, V., Lany, S., Zhang, X. \& Zunger, A. Correcting density functional theory for accurate predictions of compound enthalpies of formation: fitted elemental-phase reference energies. Phys. Rev. B 85, 115104 (2012).

\section{ACKNOWLEDGEMENTS}

K.C., K.F.G. and F.T. thank National Institute of Standards and Technology for funding, computational and data-management resources. K.C. also thank the computational support from XSEDE computational resources under allocation number (TG-DMR 190095).

\section{AUTHOR CONTRIBUTIONS}

K.C. and K.F.G. developed the workflow. K.C. carried out the DFT calculations and analyzed the DFT data. K.F.G. carried out the magnetic ordering calculations. J.J. and R.P. carried out the $G_{0} W_{0}$ based calculations. All the authors contributed to writing the manuscript.

\section{COMPETING INTERESTS}

The authors declare no competing interests.

\section{ADDITIONAL INFORMATION}

Correspondence and requests for materials should be addressed to K.C.

Reprints and permission information is available at http://www.nature.com/ reprints

Publisher's note Springer Nature remains neutral with regard to jurisdictional claims in published maps and institutional affiliations.

(i) Open Access This article is licensed under a Creative Commons Attribution 4.0 International License, which permits use, sharing, adaptation, distribution and reproduction in any medium or format, as long as you give appropriate credit to the original author(s) and the source, provide a link to the Creative Commons license, and indicate if changes were made. The images or other third party material in this article are included in the article's Creative Commons license, unless indicated otherwise in a credit line to the material. If material is not included in the article's Creative Commons license and your intended use is not permitted by statutory regulation or exceeds the permitted use, you will need to obtain permission directly from the copyright holder. To view a copy of this license, visit http://creativecommons. org/licenses/by/4.0/.

This is a U.S. government work and not under copyright protection in the U.S.; foreign copyright protection may apply 2020 